\title{
VvBAP1, a Grape C2 Domain Protein, Plays a Positive Regulatory Role Under Heat Stress
}

\author{
Qing $\mathrm{Ye}^{+}$, Jintao $\mathrm{Yu}^{\dagger}$, Zhen Zhang ${ }^{\dagger}$, Lixia Hou and Xin Liu * \\ Key Lab of Plant Biotechnology in University of Shandong Province, College of Life Science, Qingdao Agricultural University, \\ Qingdao, China
}

\section{OPEN ACCESS}

Edited by:

Andrew Wood,

Southern Illinois University

Carbondale, United States

Reviewed by:

Luisa C. Carvalho,

University of Lisbon, Portugal

Kazuo Nakashima,

Japan International Research Center

for Agricultural Sciences (JIRCAS),

Japan

${ }^{*}$ Correspondence:

Xin Liu

liuxin6080@126.com

${ }^{t}$ These authors have contributed equally to this work

Specialty section:

This article was submitted to

Plant Abiotic Stress,

a section of the journal

Frontiers in Plant Science

Received: 20 March 2020 Accepted: 11 September 2020

Published: 09 November 2020

Citation:

Ye Q, Yu J, Zhang Z, Hou L and Liu X (2020) VvBAP1, a Grape C2 Domain Protein, Plays a Positive Regulatory Role Under Heat Stress.

Front. Plant Sci. 11:544374. doi: 10.3389/fpls.2020.544374
Temperature is considered one of the critical factors directly influencing grapevine during the three primary growth and development stages: sprout, flowering, and fruit-coloring, which is strongly correlated to the yield and quality of the grape. The grapevine is frequently exposed to high-temperature conditions that are detrimental to growth. However, the mechanisms of the heat stress response and adaptation in grapevine are not adequately studied. The Arabidopsis copine gene AtBON1 encodes a highly conserved protein containing two $\mathrm{C} 2$ domains at the amino terminus, participation in cell death regulation and defense responses. Previously, we showed that a BON1 association protein from the grapevine, $V_{V} B A P 1$, plays a positive role in cold tolerance. Similarly, the involvement of $V_{V B A P} 1$ in the resistance to heat stress was also found in the present study. The results indicated $V_{V B A P} 1$ was significantly induced by high temperature, and the elevated expression of $V_{V B A P 1}$ was significantly higher in the resistant cultivars than the sensitive cultivars under heat stress. Seed germination and phenotypic analysis results indicated that overexpression of VVBAP1 improved Arabidopsis thermoresistance. Compared with the wild type, the chlorophyll content and net photosynthetic rate in VVBAP1 overexpressing Arabidopsis plants were markedly increased under heat stress. At high temperatures, overexpression of VVBAP1 also enhanced antioxidant enzyme activity as well as their corresponding gene transcription levels, to reduce the accumulation of reactive oxygen species and lipid peroxidation. Besides, the transcriptional activities of HSP70, HSP101, HSFA2, and HSFB1 in VVBAP1 overexpressing Arabidopsis plants were significantly up-regulated compare to the wild type. In summary, we propose that VVBAP1 may play a potential important role in enhanced grapevine thermoresistance, primarily through the enhancement of antioxidant enzyme activity and promoted heat stress response genes expression. 


\section{INTRODUCTION}

Temperature is considered one of the critical factors directly influence grapevine more the three primary growth and development stages: sprout, flowering and fruit-coloring (Lorenz et al., 1995). Both yield and quality of grapevine are reduced when encountered high temperatures (Wahid et al., 2007). Therefore, high temperature is one of the principal limiting factors in the development of grapevine economic industry worldwide. Through climate prediction models, the Intergovernmental Panel on Climate Change predicted an increase in global mean temperatures between $1.5^{\circ} \mathrm{C}$ and $5^{\circ} \mathrm{C}$ during the 21st century. Also, global warming will likely accompany with more frequent and powerful extreme temperature events (Lobell et al., 2008). Thus, revealing the mechanism of the grapevine thermoresistance has become a vital research topic that provides a reliable theoretical basis for grapevine breeding.

When exposed to a high-temperature conditions, a series of injuries in plants, including protein misfolding and denaturation, irreversible loss of enzyme activity, and disruption of cellular structural components, occur (Schöffl et al., 1998; Howarth, 2005). These damaging events ultimately caused a severe reduction in the net photosynthetic rate and ion flux, excessive production of reactive oxygen species (ROS), such as hydrogen peroxide $\left(\mathrm{H}_{2} \mathrm{O}_{2}\right)$ and superoxide $\left(\mathrm{O}_{2}^{-}\right)$, thus inhibition of plant growth (Bokszczanin et al., 2013). To maintain metabolic homeostasis under heat stress that the organisms can survive and even multiply, plants have formed physiological, biochemical, cellular, and molecular regulatory mechanisms to precisely regulate thermoresistance (Bartels and Sunkar, 2005; Zhuang et al., 2014).

As ROS induced by heat stress may cause oxidative damage, plants have also evolved a unique ROS scavenging systems, like superoxide dismutase (SOD), catalase (CAT), ascorbate peroxidase (APX), peroxidase (POD) and other antioxidant enzymes, might work with synergy to reduce accumulation of cellular ROS and attenuate oxidative injury to plants (Mittler et al., 2011). Additionally, more and more results have indicated that heat shock proteins (HSPs) are important molecular chaperones that ensure protein proper folding, which are essential to help plants acquire thermotolerance (Chen et al., 2011). Moreover, the small HSP (sHSP) and heat stress transcription factors (HSFs) were also reported to help plant growth and development under heat stress (Qu et al., 2013).

Grape (Vitis vinifera L.) is one of the most essential economic fruit crops throughout the world. In recent years, China has the most productive and second most widely cultivated area of grapevine worldwide. However, the fruit yield and quality of grapevine are often highly influenced by extreme climatic conditions, especially heat stress (Szenteleki et al., 2012). In many main centers of origin for grapevine, the daily maximum temperature can often surpass $40^{\circ} \mathrm{C}$, even beyond $45^{\circ} \mathrm{C}$, which has seriously limited the development of the grapevine economic industry (Salazar-Parra et al., 2010; Pillet et al., 2012). However, in elucidating the response and adaptation of grapevine to heat stress, immense efforts have been put into exploring the physiological and morphological changes. Recently, many studies have offered unique insights into understanding the heat stress responses of grapevine via transcriptomic and proteomic analyses (Kosová et al., 2011; Jiang et al., 2017). In grapevine leaves, several sHSPs and APX encoding genes identified as playing significant roles in thermotolerance (Liu et al., 2012). Previous transcriptome data have revealed high transcript levels of a series of $V v H S F$ genes such as $V v H S F A 1 a$, VaHSFA1a,VvHSFA2a,VaHSFA2a, and VvHSFB2b in Vitis vinifera or Vitis amurensis, permitting adaptation to heat stress (Liu et al., 2012; Xin et al., 2013; Rocheta et al., 2014; Xu et al., 2014). Additionally, VvHSFA2a expression is up-regulated in grapevine berries during heat stress (Pillet et al., 2012). Similarly, it has been demonstrated that the $V v H S F B 2 b$ homologous gene $V p H S F B 2 b$ is related to heat resistance in Chinese wild grape Vitis pseudoreticulata (Peng et al., 2013). Hu et al. (2016) reported ten VpHSFs, especially VpHSFB1a, VpHSFC1a, $V p H S F A 2 a, V p H S F A 3 a$, and VPHSFA6a were markedly upregulate exposed to heat stress, the results suggest that their positive regulation roles of heat stress responsive in Vitis pseudoreticulta. Furthermore, the transcription of galactinol synthase gene, VvGOLS1 was detected significantly as upregulated in grape berries under high temperature conditions (Pillet et al., 2012). Although numerous genes that relate to grapevine thermotolerance have been recognized, knowledge about the precise functions and molecular mechanism are largely unknown at the present time.

In Arabidopsis, AtBAP1 (BON1 ASSOCIATED PROTEIN 1) has been demonstrated to belong to $\mathrm{C} 2$ domain phospholipidbinding protein as a functional partner of AtBON1 (BONZAI1). AtBON1 encodes a highly conserved protein containing two C2 domains at the $\mathrm{N}$-terminus, is involved in cell death regulation and defense responses (Hua et al., 2001). Arabidopsis AtBON1deficient mutant displayed reduced plant height at the optimum growth temperature, and that overexpression of AtBAP1 could compensate for the dwarf phenotype of the bon1-1 mutant, indicating these two proteins have similar biological functions (Hua et al., 2001; Yang and Hua, 2004). Subsequent study has shown that AtBAP1 could suppress programmed cell death induced by virulent pathogens and ROS (Yang et al., 2007). Furthermore, the transcription factor AtICE1 can bind to the promoter of $A t B A P 1$, thus promoting the elevated transcription level of AtBAP1 under cold stress (Zhu et al., 2011). However, the biological role of $B A P 1$ in grapevine has not been fully elucidated.

In our previous study, we cloned and functionally characterized $V v B A P 1$ from the grapevine ' $\mathrm{F}-242$ ' as the nearest orthologue to AtBAP1 (Zhang et al., 2014). Then, we found that $V v B A P 1$ was correlated with the cold resistance in grapevine, $V v B A P 1$ could regulate the soluble sugar content and enhance antioxidant enzyme activities, thereby promoting the grape cold resistance (Hou et al., 2018). Recently, Cao et al. (2019) demonstrated that $V v B A P 1$ may functioning as an important factor in suppressing grape berries cell death, its transcript was significantly inhibited by drought stress. 
However, further studies should be conducted to examine whether $V v B A P 1$ involved in the heat tolerance of grapes. Thus, we evaluated $V v B A P 1$ expression in cultivars of grapevine that are known to exhibit different responses to heat stress conditions. Further, the VvBAP1-overexpressing Arabidopsis plants were used to analyze the physiological functions of $V v B A P 1$ by measuring a series of physiological indexes relevant to heat stress response, with an aim of revealing the $V v B A P 1$-mediated mechanisms which are inducing thermoresistance in grapes.

\section{MATERIALS AND METHODS}

\section{Plant Materials and Growth Conditions}

Shoots (with buds) of four grape (Vitis vinifera L.) cultivars named 'Chardonnay', 'Cabernet Sauvignon', 'Zuoyouhong' and 'Beta' served as explants. The shoots were rinsed with water overnight, and surface sterilized with $75 \%(\mathrm{v} / \mathrm{v})$ ethanol for $30 \mathrm{~s}$, followed by $0.1 \% \mathrm{HgCl}_{2}$ for $8 \mathrm{~min}$. After that, the explants were washed 3-5 times with sterile water. Next, a pair of scissors was used to cut approximately $2-3 \mathrm{~cm}$ of stem segment, leaving the apical bud intact. The explants were cultured on sterile MS solid medium (half strength) containing $0.57 \mu \mathrm{M}$ IAA (indole acetic acid). Culture conditions were as follows: $12 \mathrm{~h}$ light/ $12 \mathrm{~h}$ dark cycle (light intensity of $200 \mu \mathrm{mol} \cdot \mathrm{m}^{-2} \cdot \mathrm{s}^{-1}$ ); $25 \pm 1^{\circ} \mathrm{C}$. The tissue culture seedlings were used in experiments after 40-55 days.

Wild type Arabidopsis thaliana used herein was of the ecotype Columbia (Col-0). The transgenic Arabidopsis plants overexpressing $V v B A P 1$ (OEVvBAP1-38 and OEVvBAP1-40) were described in our previous study (Hou et al., 2018). Regarding plant growth, we surface sterilized the Arabidopsis seeds and maintained them at $4^{\circ} \mathrm{C}$ for $72 \mathrm{~h}$. After that, they were germinated and cultured on sterile MS solid medium at $22 \pm 1^{\circ} \mathrm{C}$ under a $16 \mathrm{~h}$ light $/ 8 \mathrm{~h}$ dark cycle (light intensity of $120 \mu \mathrm{mol} \cdot \mathrm{m}^{-2} \cdot \mathrm{s}^{-1}$ ).

\section{Heat Stress Treatment}

To test the response of $V v B A P 1$ to high temperature, the 45-55 days old grape subcultured seedlings were placed in a growth chamber at $40^{\circ} \mathrm{C}$ for $0,3,6,9,12,18$, and $24 \mathrm{~h}$. Control plants were maintained at $25 \pm 1^{\circ} \mathrm{C}$. At the end of each time point, the leaves were sampled then preserved in liquid nitrogen for RNA extraction.

Regarding Arabidopsis seed germination analysis, the seeds were maintained for $6 \mathrm{~h}$ at $45^{\circ} \mathrm{C}$ prior to germination. Seeds for each genotype were sown (300 per plate) on the same MS solid medium and maintained at $22^{\circ} \mathrm{C}$ under constant light $(60$ $\left.\mu \mathrm{mol} \cdot \mathrm{m}^{-2} \cdot \mathrm{s}^{-1}\right)$. The seeds were not received, $45^{\circ} \mathrm{C}$ treatment was used as the control. Seeds that exhibited a clear protrusion of the radicle via the seed coat were considered germinated. The number of germinated seeds were recorded after every $12 \mathrm{~h}$ during the experiment.

Seven-day old seedlings of each genotype Arabidopsis were placed at $45^{\circ} \mathrm{C}$ for $2 \mathrm{~h}$, then maintained them to continue to cultivate for $2-3$ days at $22^{\circ} \mathrm{C}$. The 4 weeks phase seedlings of each genotype Arabidopsis were treated at $42^{\circ} \mathrm{C}$ for $8 \mathrm{~h}$, then plants were recovered to grow at $22^{\circ} \mathrm{C}$ for 10 days. Then the phenotypes were observed, and the survival rates were measured. Control plants were cultured at $22^{\circ} \mathrm{C} \pm 2^{\circ} \mathrm{C}$.

The 4 weeks phase seedlings of each genotype Arabidopsis were treated at $45^{\circ} \mathrm{C}$ for $2 \mathrm{~h}$, then the change electrolyte leakage, malondialdehyde (MDA) content, SOD, POD, CAT and APX activities, expression levels of $\mathrm{Cu} / \mathrm{Zn}$ SOD, POD2, CAT1, CAT2, $C A T 3, A P X 1, A P X 2$ and heat response-related gene were tested.

\section{qRT-PCR}

We employed the CTAB method (Iandolino et al., 2004) to extract total RNA from the leaves of the experimental plants. The RNA was then reverse transcribed to cDNA using the Prime Script RT reagent Kit with the Gdna Eraser (TaKaRa, Dalian, China). The MyiQ Real-Time PCR Detection System (Bio-Rad, USA) was used to perform RT-PCR. SYBR green I (BioWhittaker Molecular Applications) was included in the reaction master mix. The following reaction conditions were used: $95^{\circ} \mathrm{C}$ for $60 \mathrm{~s} ; 40$ cycles of $95^{\circ} \mathrm{C}$ for $10 \mathrm{~s} ; 56^{\circ} \mathrm{C}$ for $20 \mathrm{~s}$; and $72^{\circ} \mathrm{C}$ for $15 \mathrm{~s}$. Each experiment containing three replicates was repeated at least thrice. Relative gene expression was determined using the $2^{-\Delta \Delta C T}$ method (Livak and Schmittgen, 2001). Genes AtACTIN2 or VvACTIN served as internal control for Arabidopsi and grape, respectively. Table S1 shows the primers used in the qRT-PCR experiments.

\section{MDA Content and Electrolyte Leakage}

MDA content was estimated by the method as described previously (Ding et al., 2007). Briefly, $0.1 \mathrm{~g}$ leaves were ground into homogenate in $1 \mathrm{ml} \mathrm{10 \% (w/v)} \mathrm{trichloroacetic} \mathrm{acid} \mathrm{(TCA),}$ and then supernatant was collected by centrifugation for $10 \mathrm{~min}$ at $4000 \mathrm{rpm}$. Next, $500 \mu \mathrm{l}$ of the supernatant was added to equal volume of $10 \%(\mathrm{w} / \mathrm{v}) \mathrm{TCA}$, containing $0.6 \%(\mathrm{w} / \mathrm{v})$ thiobarbituric acid (TBA). The mixture was then incubated at $100^{\circ} \mathrm{C}$ for $15 \mathrm{~min}$, and then centrifuged for $10 \mathrm{~min}$ at $4000 \mathrm{rpm}$ after cooling to room temperature. The absorbance of the mixture was measured at $532 \mathrm{~nm}$ then adjusted at $600 \mathrm{~nm}$ for non-specific absorbance. The quantity of MDA was computed from the extinction coefficient of $155 \mathrm{mM}^{-1} \mathrm{~cm}^{-1}$ and presented as $\mu \mathrm{mol} \mathrm{kg}{ }^{-1}$, in which one unit was equivalent to $1 \mu \mathrm{mol}$ MDA per $\mathrm{kg}$ of pulp.

Electrolyte leakage from the leaf discs were determined as per the methods described previously (Zhao et al., 2009), with a few variations. In brief, we rinsed the treated leaves with deionized (DI) water and left then to dry. Next, the leaf discs were obtained using a circular borer, and then soaked in DI water at $25^{\circ} \mathrm{C}$ for $1 \mathrm{~h}$. The electrical conductivity (EC1) of the leakage solution from the leaf discs was detected with a conductivity meter (YSI model 55). Then, the mixture was brought to a boil for $10 \mathrm{~min}$. Both total ionic strength and the electrical conductivity (EC2) were measured after cooling the solution to room temperature. The formula below was used to calculate the relative permeability of the membrane: EC1/EC2 $\times 100 \%$.

Each experiment contained three biological replicates and was repeated at least thrice.

\section{Measurement of Chlorophyll Content and Photosynthetic Rate}

A portable chlorophyll meter (SPAD-502PLUS, Minolta, Tokyo, Japan) was used to measure the chlorophyll content. The rate of 
photosynthesis was assessed using a liquid-phase oxygen measurement system (CHLOPOLAB-2, Hansatech, King's Lynn, UK), following the instructions provided by the manufacturer. While taking the measurements, plants were maintained in $200 \mu \mathrm{mol} \mathrm{m} \mathrm{m}^{-2} \mathrm{~s}^{-1}$ light intensity at $25^{\circ} \mathrm{C}$.

\section{Detection of Reactive Oxygen Species (ROS)}

Reactive oxygen species (ROS) accumulation was determined by assessing the levels of superoxide $\left(\mathrm{O}_{2}^{-}\right)$and hydrogen peroxide $\left(\mathrm{H}_{2} \mathrm{O}_{2}\right)$ via histochemical staining. We performed 3,3' diaminobenzidine (DAB) and nitro-blue tetrazolium (NBT) staining according to previous publication (Wang $\mathrm{K}$. et al., 2017). Each treatment, rosette leaves were picked and soaked in DAB ( $\left.1 \mathrm{mg} \mathrm{mL}^{-1}, \mathrm{pH} 3.8\right)$ or NBT solution $\left(0.1 \mathrm{mg} \mathrm{mL}^{-1}\right)$ at $25^{\circ} \mathrm{C}$ for $8 \mathrm{~h}$, and then photographed and subjected to analysis after sufficient bleaching in boiling $75 \%(\mathrm{v} / \mathrm{v})$ ethanol. The quantitative measurements of $\mathrm{H}_{2} \mathrm{O}_{2},\left(\mathrm{O}_{2}^{-}\right)$were performed according to Zhang et al. (2011). At least three experiments were performed, each experiment contained three biological replicates.

\section{Measurement of Antioxidant Enzyme Activity}

The frozen leaves samples ( $0.6 \mathrm{~g})$ were ground into homogenate in ice-cooled $0.1 \mathrm{M}$ phosphate buffer ( $\mathrm{pH} 7.6$ ) containing $0.5 \mathrm{mM}$ EDTA, and then supernatant was obtained by centrifugation at $12,000 \mathrm{rpm}$ for $10 \mathrm{~min}$ at $4^{\circ} \mathrm{C}$. The supernatant was collected to determinate antioxidant enzymes activities. CAT activity was measured by the previously reported protocol (Aebi, 1984), and absorbance was taken at $240 \mathrm{~nm}$. The activity of SOD and POD were investigated according to the Liang et al. (2015) method with minor changes. The activity of APX was evaluated as per the methods described previously (Wang L. et al., 2017). At least three experiments were performed, each experiment was performed in biological triplicate. The result from one set of experiments is provided here.

\section{Statistical Analysis}

Statistical analysis for all experiments were performed using SAS. Differences between multiple treatments were analyzed using one-way ANOVA and means separated by Tukey's HSD test $(\mathrm{P}<$ 0.05). Data are mean values of three independent biological replicates $\pm \mathrm{SE}$.

\section{RESULTS}

\section{Expression Profiles of $V v B A P 1$ in Grape Cultivars Leaves With Different Thermotolerance}

In order to evaluate the potential role for $V v B A P 1$ in the grapevine resistance to heat stress, we first detected the distinction between $V v B A P 1$ expression profiles in the grapevine varieties with different thermotolerance. The transcript level of $V v B A P 1$ in the leaves of resistant cultivars 'Zuoyouhong' and 'Beta' were markedly higher compare to the other two sensitive grapevine cultivars 'Cabernet Sauvignon' and 'Chardonnay' under non-stressed conditions. After heat stress, the $V v B A P 1$ transcript level in all grapevine cultivars increased significantly, and $V v B A P 1$ from 'Zuoyouhong' and 'Beta' were more sensitive to high temperature, as its transcript level increased much higher compare to that from 'Cabernet Sauvignon' and 'Chardonnay' (Figure 1A). In the follow-up experiment, we further used the tissue culture seedlings of the sensitive cultivars 'Chardonnay' and the resistant cultivar 'Zuoyouhong' to analyze the expression pattern of $V v B A P 1$ with $40^{\circ} \mathrm{C}$ treatment. The results indicated $V v B A P 1$ was significantly induced in the two grapevine cultivars by hightemperature, showing the highest transcriptional expression at $9 \mathrm{~h}$. Besides, the transcript level of $V v B A P 1$ in 'Zuoyouhong' was always significantly higher than that in 'Chardonnay' (Figure 1B). These data suggest that $V v B A P 1$ could play an essential function in heat stress response in the grapevine.

A

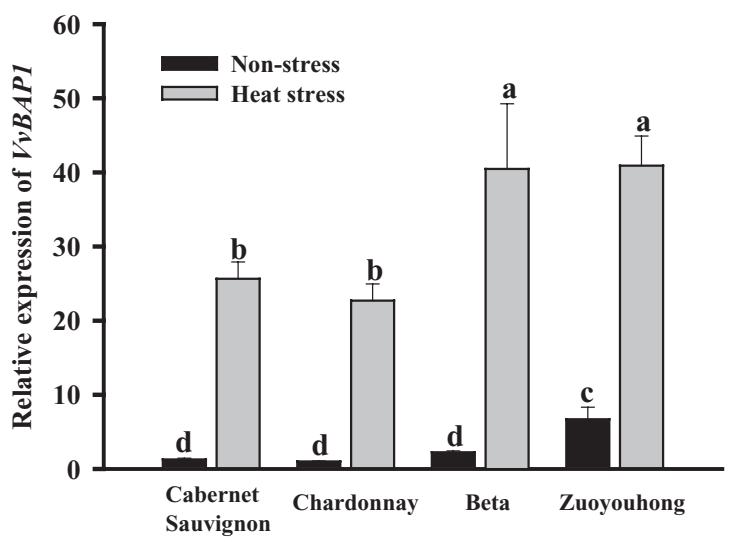

B

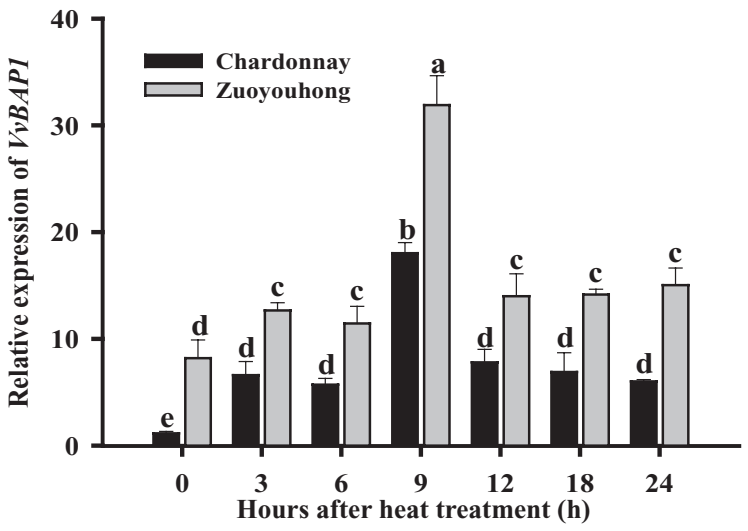

FIGURE 1 | Expression profiles of VVBAP1. (A) Relative transcript level of VVBAP1 in leaves of different grape varieties with $40^{\circ} \mathrm{C}$ treatment after $9 \mathrm{~h}$. Three independent experimental replications were conducted. Values are the means $\pm \mathrm{SE}$ of three independent experiments $(P<0.05)$. (B) Expression of $V_{V B A P 1}$ in the sensitive grapevine cultivars 'Chardonnay' and the resistant varieties 'Zuoyouhong' with $40^{\circ} \mathrm{C}$ treatment. Three independent experimental replications were conducted. Values are the means \pm SE of three independent experiments $(P<0.05)$. Lowercase letters above bars denote significant differences attested by Tukey's HSD test. 


\section{Effect Of VvBAP1 Overexpression on the Growth of Transgenic Arabidopsis Plants Under Heat Stress}

In order to further analyze the physiological function of $V v B A P 1$ in the heat stress tolerance, the $V v B A P 1$-overexpressing Arabidopsis plants, which have been reported in our previous research (Hou et al., 2018), were used for seed germination and phenotypic analysis under heat stress. The results of the seed germination rate showed that there was no substantial difference between the seeds of each genotype under non-stressed conditions (Figure 2A), when germinated after heat stress treatment, all plants displayed a significant reduction in seed germination percentages, and the two ectopic overexpressing lines showed faster germination rate compared to the wild type
A

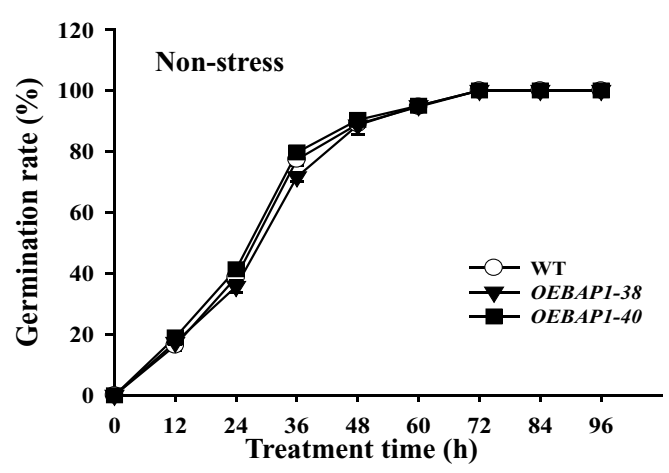

C

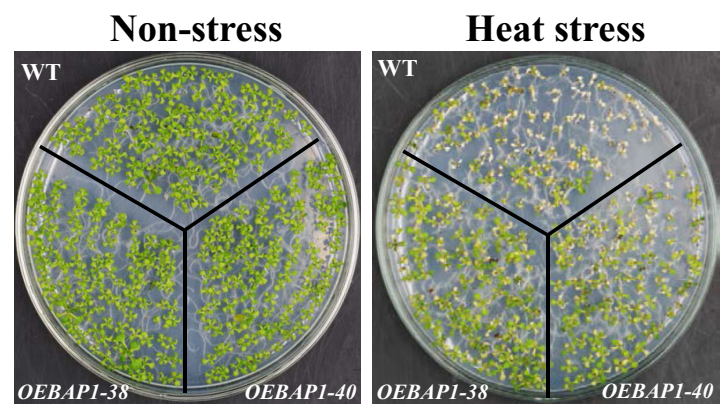

E

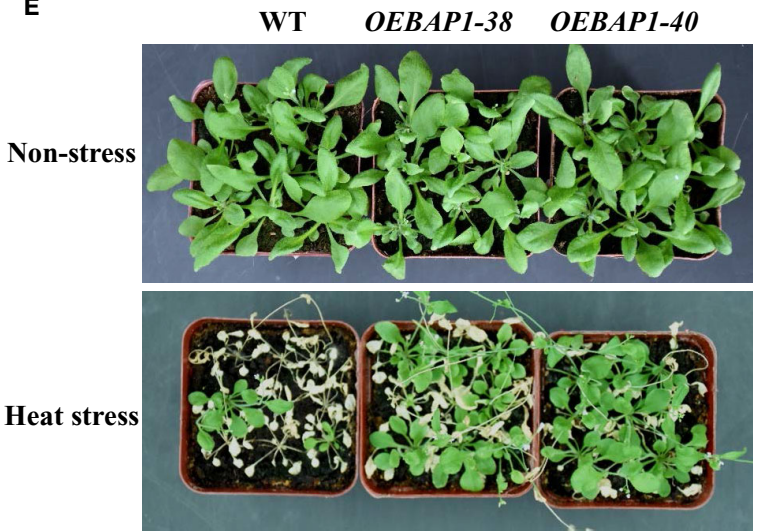

B

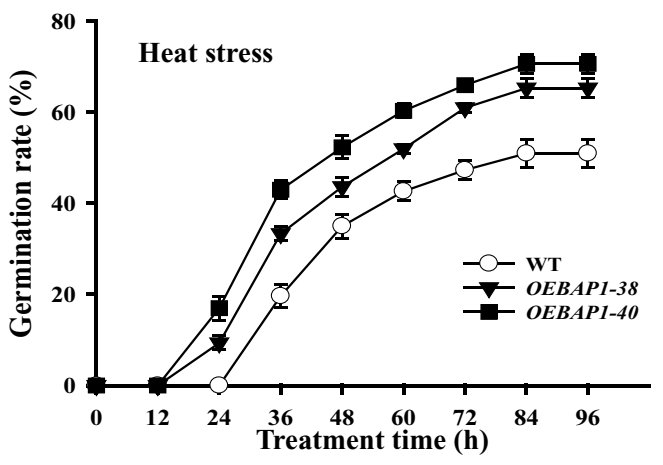

D

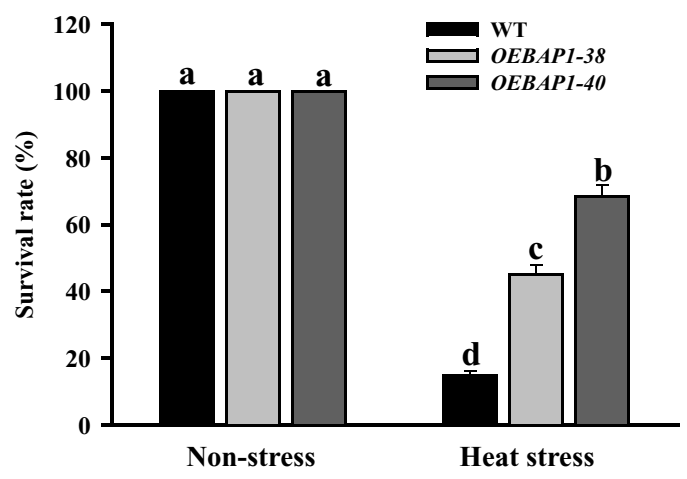

$\mathbf{F}$

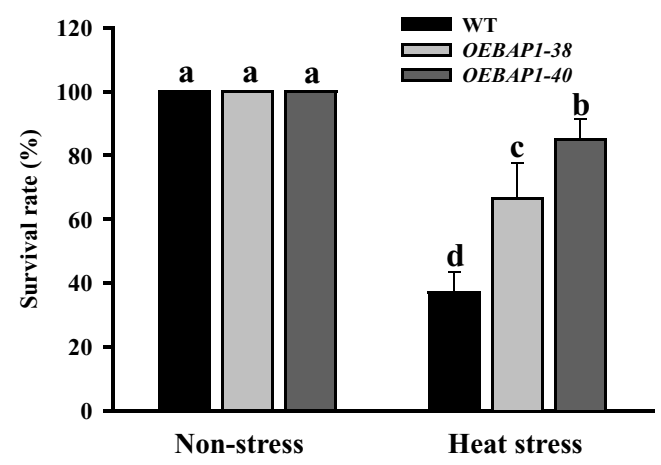

FIGURE 2 | Effects of high temperature on the growth of VVBAP1-overexpressing Arabidopsis plants. Time course of seed germination rates for the wild type and three independent transgenic Arabidopsis plants under a normal temperature (A) and after heat stress (B). Each experiment was conducted at least thrice and more than 300 seeds were measured in each replicate. Values are the means \pm SE of three independent experiments $(P<0.05)$. The phenotype (C) and survival rates (D) of the wild type and three independent transgenic Arabidopsis seedlings under heat stress $\left(45^{\circ} \mathrm{C}\right.$ for $\left.6 \mathrm{~h}\right)$. The phenotype (E) and survival rates (F) of the adult wild type and three independent transgenic Arabidopsis seedlings under heat stress $\left(42^{\circ} \mathrm{C}\right.$ for $\left.8 \mathrm{~h}\right)$. Each experiment was conducted thrice. Values are the means $\pm \mathrm{SE}$ of three independent experiments $(P<0.05)$. Lower-case letters above bars denote significant differences attested by Tukey's HSD test. 
(Figure 2B). When grown under non-stressed conditions, all the plants exhibited similar phenotypes. However, after heat treatment, the $V v B A P 1$-overexpressing Arabidopsis exhibited better growth than wild type plants (Figures $2 \mathbf{C}, \mathbf{E}$ ), and the transgenic lines displayed obviously higher survival rates compared to the wild type (Figures 2D, F). These results indicated that $V v B A P 1$ did indeed improve resistance to heat stress in plants.

\section{Effects of VvBAP1 Overexpression on Physiological Indexes of Transgenic Arabidopsis Plants Under Heat Stress}

To further investigate the function of $V v B A P 1$ in regulating thermoresistance, the electrolyte leakage and MDA content in leaves of $V v B A P 1$-overexpressing Arabidopsis and the wild type was analyzed. We found that there was no apparent differences in the electrolyte leakage and MDA content between each genotype plants under non-stressed conditions. However, all the plants exhibited a remarkable increase in the MDA content and electrolyte leakage following heat treatment, and this was more significant in wild type, relative to the transgenic plants (Figures 3A, B). These findings demonstrated that $V v B A P 1$ has a positive role in improving the cytomembrane stability to enhance heat resistance in plants further.

Studies have shown the plant photosynthesis can be suppressed early following exposure to high temperature
(Larkindale et al., 2005; Allakhverdiev et al., 2008). Given this, we explored the impact of heat stress on chlorophyll content and photosynthetic rate. As shown in Figure 3C, under non-stressed conditions, transgenic lines exhibited marginally higher chlorophyll content, relative to the wild type plants. Heat stress exerted no substantial impact on the transgenic plants chlorophyll content, but dramatically reduced that of the wild type. The photosynthetic rate of each genotype of plants declined after heat stress treatment. However, the photosynthetic rate of $V v B A P 1$-overexpressing lines was considerably higher compared to the wild type (Figure 3D). These provide further evidence that the photosynthetic ability of plants overexpressing $V v B A P 1$ was less affected when compared with the wild type under hightemperature condition, which could be a reason for the $V v B A P 1$ mediated increase tolerance to heat stress.

\section{Changes in the Levels of Accumulated ROS in VvBAP1-Overexpressing Arabidopsis Leaves Under Heat Stress.}

When plants are subjected to heat stress, oxidative damage caused by excessive of ROS production was identified as a critical limiting factor in plant growth by disrupting macromolecules and cytomembrane (Miller et al., 2007; Larkindale and Vierling, 2008). Consequently, the effects of heat stress on the contents of $\mathrm{H}_{2} \mathrm{O}_{2}$ and $\left(\mathrm{O}_{2}^{-}\right)$were measured by the histochemical detection in the leaves of each genotype
A
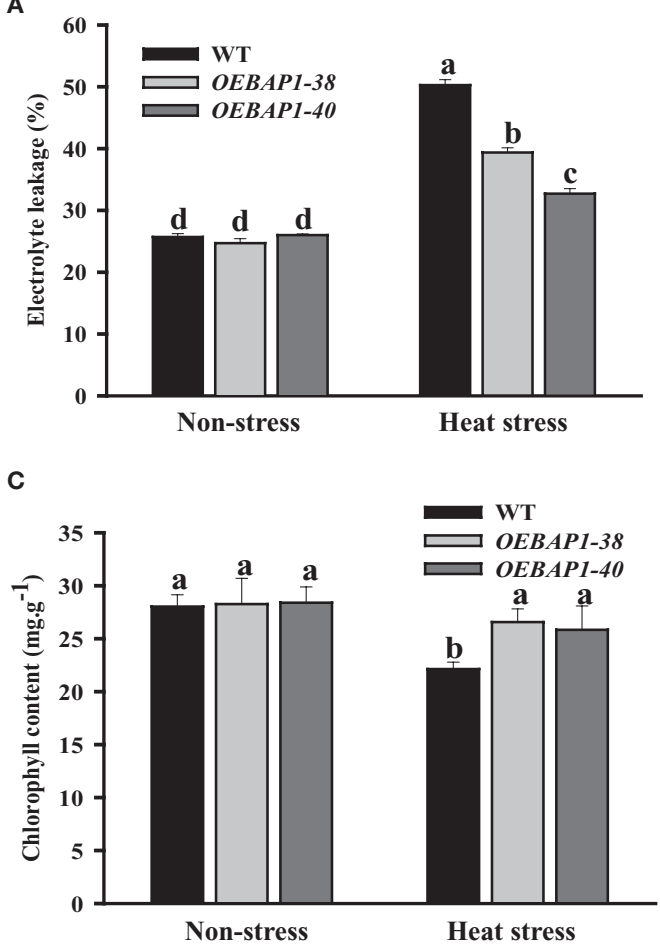

B

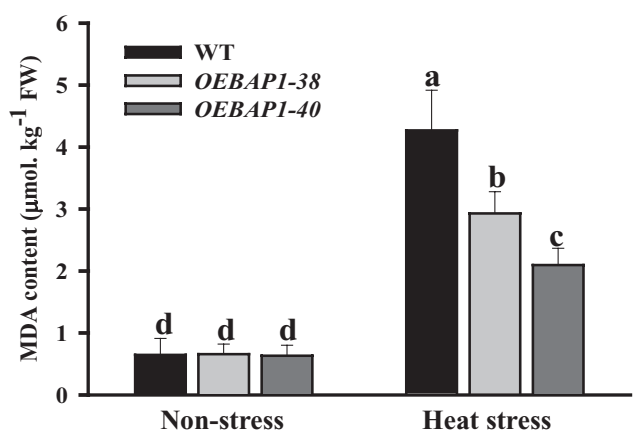

D

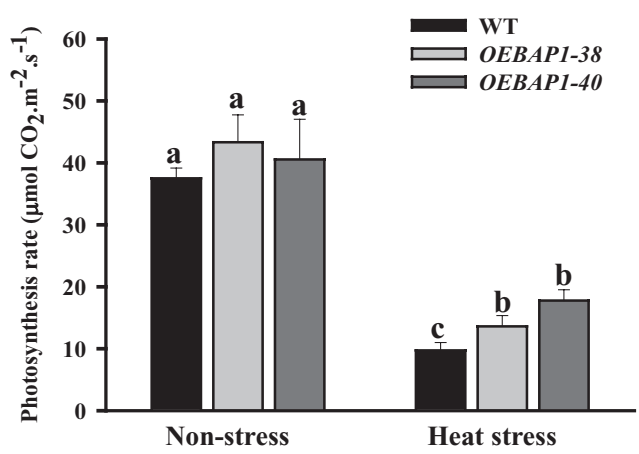

FIGURE 3 | Analysis of physiological indexes of VVBAP1-overexpressing Arabidopsis under heat stress. The electrolyte leakage (A), MDA content (B), chlorophyll content (C), photosynthetic rate (D) in the leaves of overexpressing lines under heat stress $\left(45^{\circ} \mathrm{C}\right.$ for $\left.2 \mathrm{~h}\right)$. Each experiment was conducted thrice. Values are the means \pm SE of three independent experiments $(P<0.05)$. Lower-case letters above bars denote significant differences attested by Tukey's HSD test. 
plants. When stained separately with DAB and NBT, which were applied to evaluate $\mathrm{H}_{2} \mathrm{O}_{2}$ and $\left(\mathrm{O}_{2}^{-}\right)$accumulation, the leaves were similarly and lightly stained under non-stressed condition. In contrast, the leaves of the wild type displayed more intense brown coloration or blue patches, relative to the leaves of transgenic plants after heat stress (Figures 4A, B). These results showed that high temperature led to increased ROS production such as $\mathrm{H}_{2} \mathrm{O}_{2}$ and $\left(\mathrm{O}_{2}^{-}\right)$, while those in transgenic plants were significantly lower, relative to the wild type. These findings were confirmed further by conducting quantitative assays (Figures 4C, D).

\section{Overexpression of VvBAP1 Enhanced the Activities of ROS-Scavenging Under Heat Stress}

As is well known, the measurement of the activity of ROSscavenging enzymes has been extensively applied to analyze the resistance to stress in plants (Suzuki et al., 2011).The main antioxidant enzymes include SOD, POD, CAT and APX could work together to reduce cellular ROS accumulation and attenuate oxidative injury to plants (Xu et al., 2016). Thus, we assessed the activity of these four enzymes, and the relative expression of their corresponding genes $\mathrm{Cu} / \mathrm{Zn} \mathrm{SOD}, \mathrm{POD} 2$, CAT1, CAT2, CAT3, APX1 and APX2. The results showed that heat stress increased antioxidant activities and up-regulate transcription of their corresponding genes in all plants. Compared with the wild type, overexpression of $V v B A P 1$ enhanced the increase in antioxidant activities well as their corresponding genes transcription levels induced by heat stress (Figures 5A-K). Collectively, these data suggested that overexpress $V v B A P 1$ enhanced the antioxidant enzyme activity by promoting the expression of genes encoding those enzymes in vivo, resulting in reduced levels of ROS under heat stress

\section{VvBAP1 Is Involved in Heat Tolerance by Enhancing Heat Response-Related Gene Expression}

Apart from the antioxidant system, HSPs, often regarded as important molecular chaperones that ensure proper protein folding, which is vital in the growth and development of plants under the high-temperature conditions (Hahn et al., 2012). Moreover, HSFs are related to the direct regulation the
A

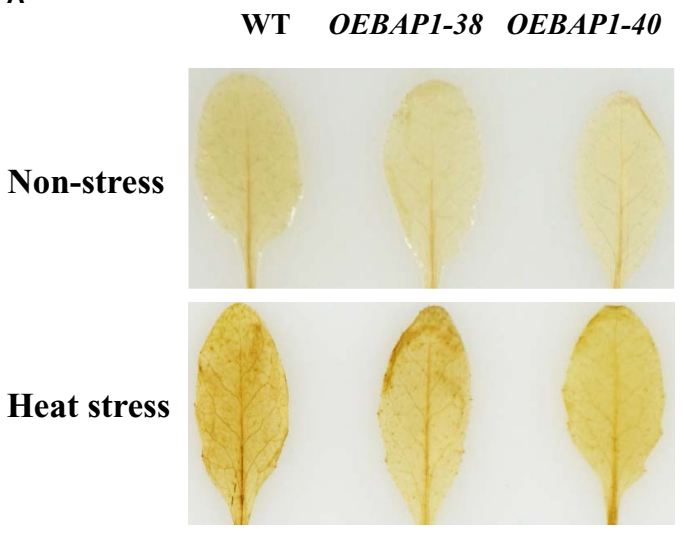

C

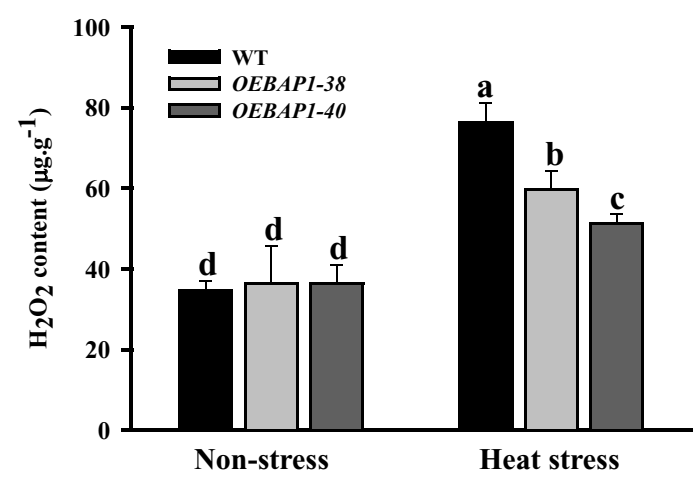

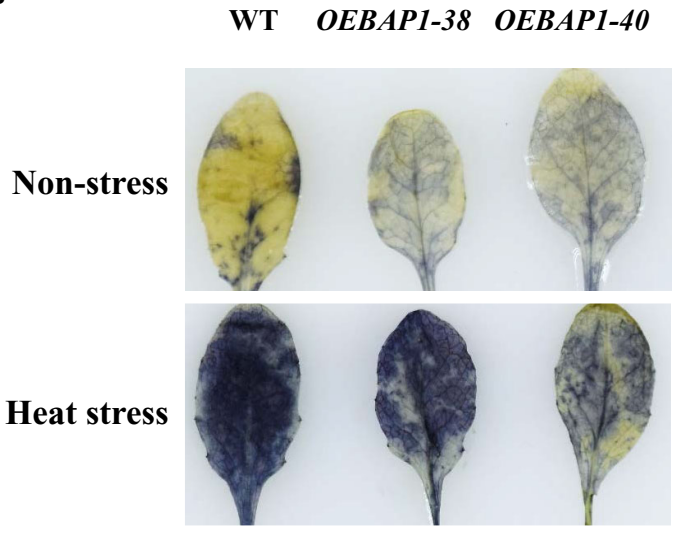

D

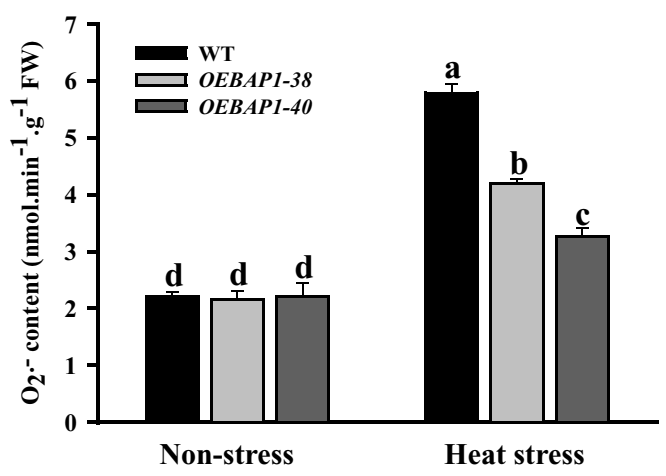

FIGURE 4 | Changes in the levels of accumulated ROS in VVBAP1-overexpressing Arabidopsis leaves under heat stress. In situ accumulation of $\mathrm{H}_{2} \mathrm{O}_{2}(\mathbf{A})$ and $\left(\mathrm{O}_{2}^{-}\right)$(B) in leaves treated with and without heat stress $\left(45^{\circ} \mathrm{C}\right.$ for $\left.2 \mathrm{~h}\right)$ revealed by DAB and NBT staining, respectively. Quantitative measurement of $\mathrm{H}_{2} \mathrm{O}_{2}(\mathbf{C})$ and $\left(\mathrm{O}_{2}^{-}\right)(\mathbf{D})$ concentrations in leaves treated with and without high temperature with and without heat stress $\left(45^{\circ} \mathrm{C}\right.$ for $\left.2 \mathrm{~h}\right)$. Each experiment was conducted thrice. Values are the means \pm SE of three independent experiments $(P<0.05)$. Lower-case letters above bars denote significant differences attested by Tukey's HSD test. 

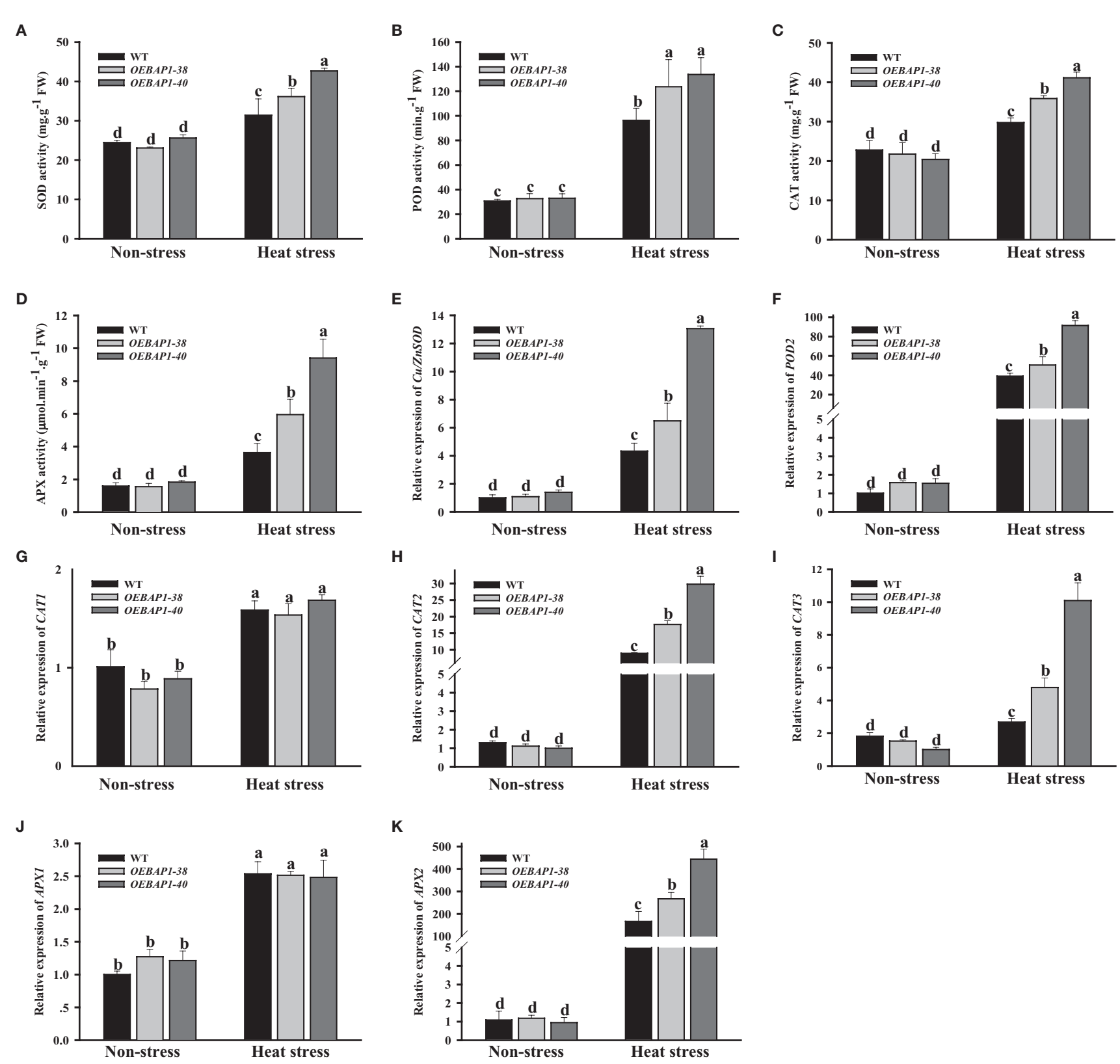

$\mathbf{K}$

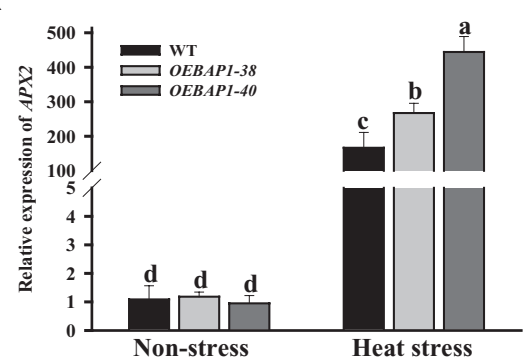

FIGURE 5 | Effects of heat stress on ROS-scavenging enzymes of VVBAP1-overexpressing Arabidopsis leaves. The activity of CAT (A), POD (B), CAT (C) and APX (D), the expression of SOD1 (E), POD2 (F), CAT1 (G), CAT2 (H), CAT3 (I), APX1 (J) and APX2 (K) in the leaves of overexpressing lines under heat stress (45 ${ }^{\circ} \mathrm{C}$ for 2 h). Each experiment was conducted thrice. Values are the means \pm SE of three independent experiments $(P<0.05)$. Lower-case letters above bars denote significant differences attested by Tukey's HSD test.

transcriptional level of heat stress-induced genes (Baniwal et al., 2004). We assessed the changes in the transcript abundances of HSP70, HSP101, HSFA2, HSFB1, and HSFB2a. After heat treatment, these genes were markedly up-regulate in all genotype plants, especially HSP70, HSP101, and HSFA2. Their transcript level was induced hundreds of times. Additionally, the transcript abundances of HSP70, HSP101, HSFA2, and HSFB1 in transgenic plants were much higher compared to the wild type (Figures 6A-E). These results suggested that the increased HSPs and HSFs transcription might be relevant to $V v B A P 1$-mediated heat stress response in grapevine, which may have improved the transgenic plants thermoresistance.

\section{DISCUSSION}

Temperature is considered as one of the critical factors directly influencing grapevine during the three primary growth and 
A

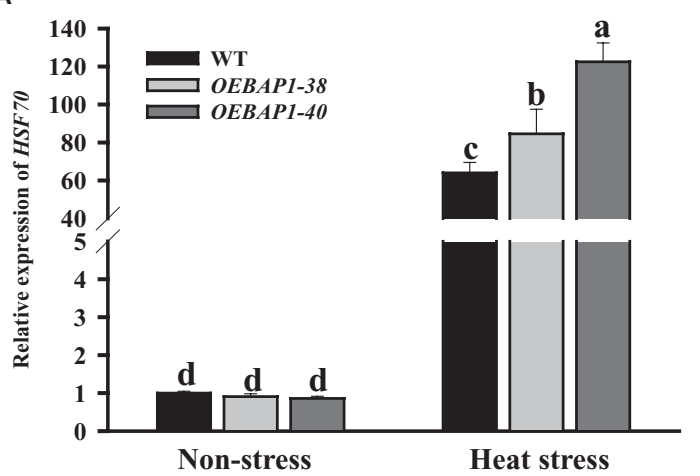

C

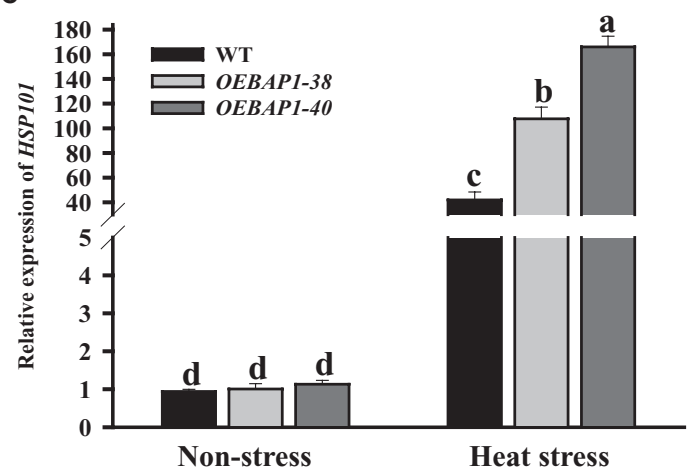

E

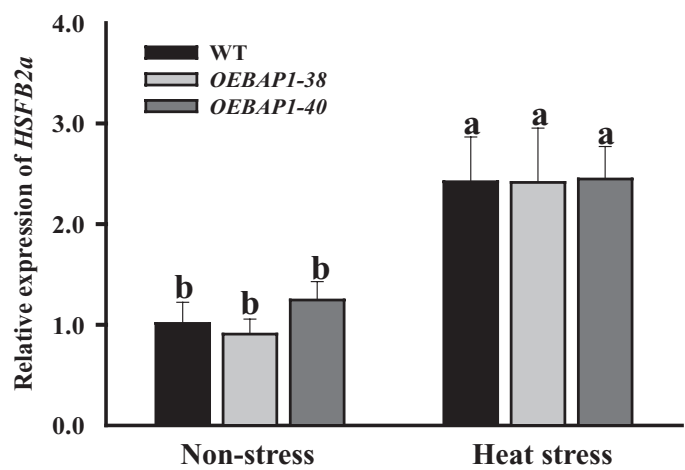

B

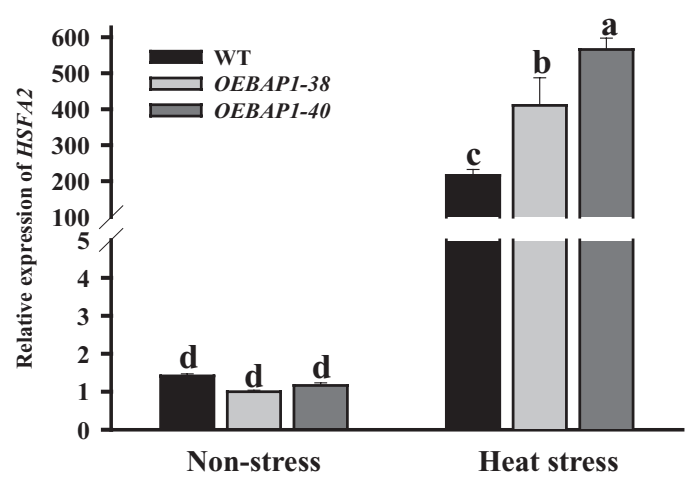

D

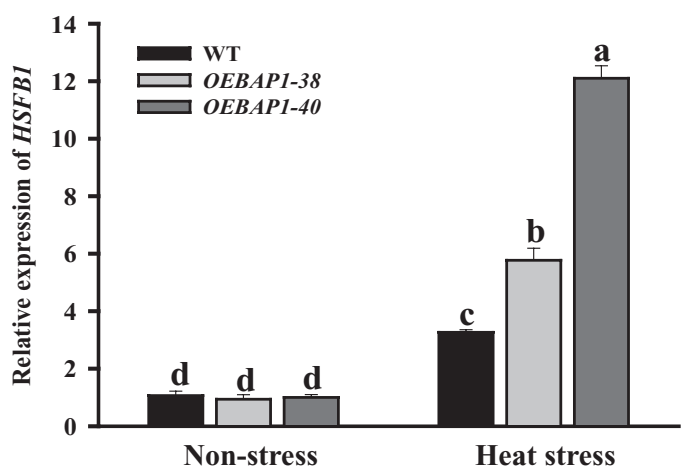

FIGURE 6 | Effects of heat stress on the expression of heat-related genes in VVBAP1-overexpressing Arabidopsis leaves. The expression of HSP70 (A), HSP101 (B), HSAFA2 (C), HSAFB1 (D) and HSFB2a (E) in the leaves of overexpressing lines under heat stress $\left(45^{\circ} \mathrm{C}\right.$ for $\left.2 \mathrm{~h}\right)$. Each experiment was conducted thrice. Values are the means \pm SE of three independent experiments $(P<0.05)$. Lower-case letters above bars denote significant differences attested by Tukey's HSD test.

development stages: sprout, flowering, and fruit-coloring (Lorenz et al., 1995), which are strongly correlated to the yield and quality of grape (Bonnefoy et al., 2013; Bonada and Sadras, 2015; Fraga et al., 2016). However, the grapevine is frequently exposed to a high-temperature condition detrimental to growth (Pereira et al., 2014). Thus, revealing the mechanism of the grapevine thermoresistance is a vital research topic that provides a reliable theoretical basis for grapevine breeding. Increasing data on transcriptomic and proteomic analyses have provided neoteric insights for elucidating the potential molecular mechanism of the grapevine thermoresistance (Liu et al., 2012; Carbonell-Bejerano et al., 2013; Liu et al., 2014; George et al.,
2015). However, understanding their precise functions and molecular mechanism in heat resistance of those genes and protein remains clarified at the present time. In this study, the underlying physiological and molecular mechanisms of $V v B A P 1$ from grapevine were preliminarily explored in response to heat stress.

BAP1 has been demonstrated to belong to C2 domain phospholipid-binding protein; it could suppress programmed cell death in Arabidopsis induced by virulent bacterial or oomycete pathogens (Yang et al., 2006; Yang et al., 2007). Later study has shown that low temperatures could induce the AtBAP1 transcription level elevate in Arabidopsis 
(Zhu et al., 2011). To reveal the biological functions of $V v B A P 1$ in grapevines, We cloned $V v B A P 1$ from the resistant grapevine cultivar 'F-242'and functionally characterized it as the nearest orthologous to AtBAP1 in our previous reports (Zhang et al., 2014). Then, we found that $V v B A P 1$ was correlated with the cold response process in grapevines, $V v B A P 1$ could regulate the soluble sugar content and enhance antioxidant enzyme activities, thereby promoting the grape cold resistance (Hou et al., 2018). In the research presented here, we showed that the elevated expression of $V v B A P 1$ was higher in the resistant cultivars compare to that from sensitive cultivars under high temperature conditions (Figure 1A). Furthermore, The qRT-PCR results indicated $V v B A P 1$ was significantly induced by hightemperature; the highest level of expression was shown at $9 \mathrm{~h}$ (Figure 1B). These data imply that $V v B A P 1$ probably positively affects the grapevine heat stress response. In the follow-up experiment, we further used the $V v B A P 1$-overexpressing Arabidopsis plants to analyze the physiological functions of VvBAP1 in heat stress response. Seed germination and phenotypic analysis results indicated that overexpression of $V v B A P 1$ improved Arabidopsis thermoresistance (Figures 2A-F).

As is well known, the measurement of electrolyte leakage contents in leaves has been extensively applied to analysis plant cell membrane damage under abiotic stresses (Moore and Roberts, 1998). MDA, the end lipid peroxidation product induced by ROS, are widely used as a marker of ROS-mediated injuries for plants (Bajji et al., 2002). The contents of chlorophyll have been commonly utilized to index the heat stress impact on photosynthesis in plants (Zhou et al., 2014; Wang X. L. et al., 2017). Thus, we measured electrolyte leakage, MDA content, chlorophyll contents, and net photosynthetic rate to evaluate the role of $V v B A P 1$ in the transgenic Arabidopsis plants under heat stress. The results found that the wild type showing obviously higher in the cell electrolyte leakage and MDA content compared to the transgenic plants, indicating the positive effects of $V v B A P 1$ especially on heat resistance in plants by improving the stability of the cytomembrane (Figures 3A, B). Additionally, the decreases of chlorophyll concentration and photosynthetic rate in transgenic plants leaves were not as apparent as in the wild type leaves under heat stress (Figures 3C, D).

Oxidative damage caused by excessive ROS production was identified as one of the principal limiting factors in plant growth under high temperatures (Hossain, 2015). Previous research has found that increased ROS levels in heat-sensitive rice are significantly more evident compared to the resistant rice under the high-temperature conditions, indicating that ROS accumulation is closely related to thermoresistance in plants (Zhao et al., 2018). In this study, high temperature led to increased ROS production such as $\mathrm{H}_{2} \mathrm{O}_{2}$ and $\left(\mathrm{O}_{2}^{-}\right)$, while those in transgenic plants markedly decreased, relative to the wild type (Figures 4A-D). These data suggested that overexpressing $V v B A P 1$ suppressed ROS excessive accumulation, which contributed to mitigating oxidative injury to plants caused by heat stress. SOD, POD, CAT, and APX have been recognized as important ROS scavengers to play a crucial function in heat stress response, their activity levels are directly associated with the acquisition of the thermotolerance in plants (Haider et al., 2017). For instance, the mutants that lacked the capacity to eliminate ROS were significantly weaker in basal thermotolerance (Larkindale et al., 2005). In addition, overexpression of TaFBA1 enhancedtransgenic tobacco basal thermotolerance by improving antioxidant enzyme activity and reducing accumulation of ROS (Li et al., 2018). Similarly, the antioxidant enzyme-encoding genes have been extensively applied to analyze ROS responsive and oxidative stress. In this study, we also found that compared with the wild type, overexpression of $V v B A P 1$ enhanced the increase in antioxidant activities well as their corresponding gene transcription levels induced by heat stress (Figures 5A-K). The results suggested that overexpression of $V v B A P 1$ increased the antioxidant enzyme activity by promoting the expression of genes encoding those enzymes in vivo, leading to reduced ROS level, resulting in enhanced plant thermoresistance.

HSPs, often regarded as critical molecular chaperones that ensure proper protein folding, are essential to help plants acquire thermotolerance under the high-temperature conditions (Hahn et al., 2012). For example, HSP70 is significantly induced by high temperatures in grapevines (Morrell et al., 1997; Zhang et al., 2005). Besides, HSFs are related to the direct regulation the transcriptional level of heat stress-induced genes (Baniwal et al., 2004). It was previously reported that $V v H s f A 1 a, V v H s f A 2 a$, $V v H s f B 1$, and $V v H s f B 2 A$ were markedly up-regulated in Vitis vinifera $\mathrm{L}$ during heat stress (Xin et al., 2013; Rocheta et al., 2014). Therefore, we detected the HSP70, HSP101, HSFA2, HSFB1 and HSFB2a expression in transgenic plants. The results of qRT-PCR revealed that the transcript abundances of HSP70, HSP101, HSFA2, and HSFB1 in VvBAP1 overexpressing Arabidopsis plants were markedly up-regulated compare to the wild type (Figures 6A-E). These data suggested that the increased HSPs and HSFs transcription might be relevant to $V v B A P 1$-mediated heat stress response in grapevine.

In summary, we propose that $V v B A P 1$ may play a potentially important role in enhanced grapevine thermoresistance, mainly by a combination of increased antioxidant enzyme activity and promoted heat stress response genes expression. Heat stress has been shown to induce $\mathrm{Ca}^{2+}$ accumulation, in order to regulate HSPs transcription and plant thermotolerance (Zhang et al., 2009; Liu et al., 2010). Whether $\mathrm{Ca}^{2+}$ regulates the affinity of VvBAP1 binding to its phospholipids substrate and therefore is involved in the heat stress response signaling network, will be necessary to investigate in follow-up experiments. In our previous study, the $V v B A P 1$ promoter has the MBS element binding with the MYB transcription factor (Hou et al., 2018). The MYB family is one of most crucial transcription factor families involved in regulating the physiological process such as development, metabolism, and stress response in plants (Dubos et al., 2010). There is a need to conduct further studies to determine whether MYB transcription factors involved in response to heat stress in grapevine. If so, can MYB proteins act as regulators and directly bind to the MBS element within $V v B A P 1$ promoter to enhance $V v B A P 1$ expression? These research questions will form the subject of future studies. 


\section{DATA AVAILABILITY STATEMENT}

The raw data supporting the conclusions of this article will be made available by the authors, without undue reservation.

\section{AUTHOR CONTRIBUTIONS}

QY performed experiments, interpreted data, and wrote the article. JY and ZZ performed experiments and interpreted the data. XL conceived and designed experiments and edited the article. LH analyzed the data. All authors contributed to the article and approved the submitted version.

\section{REFERENCES}

Aebi, H. (1984). Catalase in vitro. Method Enzymol. 105, 121-126. doi: 10.1016/ S0076-6879(84)05016-3

Allakhverdiev, S.II, Kreslavski, V. D., Klimov, V. V., Los, D. A., Carpentier, R., and Mohanty, P. (2008). Heat stress: an overview of molecular responses in photosynthesis. Photosynth. Res. 98, 541-550. doi: 10.1007/s11120-008-9331-0

Bajji, M., Kinet, J. M., and Lutts, S. (2002). The use of the electrolyte leakage method for assessing cell membrane stability as a water stress tolerance test in durum wheat. Plant Growth Regul. 36, 61-70. doi: 10.1023/a:1014732714549

Baniwal, S. K., Bharti, K., Chan, K. Y., Fauth, M., Ganguli, A., Kotak, S., et al. (2004). Heat stress response in plants: a complex game with chaperones and more than twenty heat stress transcription factors. J. Biosci. 29, 471-487. doi: 10.1007/BF02712120

Bartels, D., and Sunkar, R. (2005). Drought and salt tolerance in plants. Crit. Rev. Plant Sci. 24, 23-58. doi: 10.1080/07352680590910410

Bokszczanin, K. L., Solanaceae Pollen Thermotolerance Initial Training Network (SPOT-ITN) C, and Fragkostefanakis, S. (2013). Perspectives on deciphering mechanisms underlying plant heat stress response and thermotolerance. Front. Plant Sci. 4:315. doi: 10.3389/fpls.2013.00315

Bonada, M., and Sadras, V. O. (2015). Review: critical appraisal of methods to investigate the effect of temperature on grapevine berry composition. Aust. J. Grape Wine Res. 21, 1-17. doi: 10.1111/ajgw.12102

Bonnefoy, C., Quenol, H., Bonnardot, V., Barbeau, G., Madelin, M., Planchon, O., et al. (2013). Temporal and spatial analyses of temperature in a French wine-producing area: the Loire Valley. Int. J. Climatol. 33, 1849-1862. doi: 10.1002/joc.3552

Cao, S. F., Xiao, Z. Y., Jiranek, V., and Tyerman, S. D. (2019). The VvBAP1 gene is identified as a potential inhibitor of cell death in grape berries. Funct. Plant Biol. 46, 428-442. doi: 10.1071/FP18272

Carbonell-Bejerano, P., Santa María, E., Torres-Pérez, R., Royo, C., Lijavetzky, D., Bravo, G., et al. (2013). Thermotolerance responses in ripening berries of Vitis vinifera L. cv Muscat Hamburg. Plant Cell Physiol. 54, 1200-1216. doi: 10.1093/ pcp/pct071

Chen, B., Retzlaff, M., Roos, T., and Frydman, J. (2011). Cellular strategies of protein quality control. Cold Spring Harb Perspect. Biol. 3, a004374. doi: 10.1101/ cshperspect.a004374

Ding, Z. S., Tian, S. P., Zheng, X. L., Zhou, Z. W., and Xu, Y. (2007). Responses of reactive oxygen metabolism and quality in mango fruit to exogenous oxalic acid or salicylic acid under chilling temperature stress. Physiol. Plant 130, 112121. doi: 10.1111/j.1399-3054.2007.00893.x

Dubos, C., Stracke, R., Grotewold, E., Weisshaar, B., Martin, C., and Lepiniec, L. (2010). MYB transcription factors in Arabidopsis. Trends Plant Sci. 15, 573581. doi: 10.1016/j.tplants.2010.06.005

Fraga, H., Santos, J. A., Moutinho-Pereira, J., Carlos, C., Silvestre, J., Eiras-Dias, J., et al. (2016). Statistical modelling of grapevine phenology in Portuguese wine regions: observed trends and climate change projections. J. Agric. Sci. 154, 795811. doi: 10.1017/s0021859615000933

George, I. S., Pascovici, D., Mirzaei, M., and Haynes, P. A. (2015). Quantitative proteomic analysis of Cabernet Sauvignon grape cells exposed to thermal stresses reveals alterations in sugar and phenylpropanoid metabolism. Proteomics. 15, 3048-3060. doi: 10.1002/pmic.201400541

\section{FUNDING}

This research was funded by "National Key Research and Development Program of China (Grant No. 2018YFD1000302)" and "Natural Science Foundation of China (Grant No. 31572107 and 31872082)".

\section{SUPPLEMENTARY MATERIAL}

The Supplementary Material for this article can be found online at: https://www.frontiersin.org/articles/10.3389/fpls.2020. 544374/full\#supplementary-material

Hahn, A., Bublak, D., Schleiff, E., and Scharf, K. (2012). Crosstalk between Hsp90 and Hsp70 chaperones and heat stress transcription factors in tomato. Plant Cell. 23, 741-755. doi: 10.1105/tpc.110.076018

Haider, M. S., Kurjogi, M. M., Fiaz, M., Pervaiz, T., Jiu, S., Jia, H. F., et al. (2017). Grapevine immune signaling network in response to drought stress as revealed by transcriptomic analysis. Plant Physiol. Biochem. 121, 187-195. doi: 10.1016/ j.plaphy.2017.10.026

Hossain, M. A. (2015). Hydrogen peroxide priming modulates abiotic oxidative stress tolerance: insights from ROS detoxification and scavenging. Front. Plant Sci. 6, 420. doi: 10.3389/fpls.2015.00420

Hou, L. X., Zhang, G. K., Zhao, F. G., Zhu, D., Fan, X. X., Zhang, Z., et al. (2018). VvBAP1 is involved in cold tolerance in Vitis vinifera L. Front. Plant Sci. 9, 726. doi: $10.3389 /$ fpls.2018.00726

Howarth, C. J. (2005). "Genetic improvements of tolerance to high temperature," in Abiotic stresses: plant resistance through breeding and molecular approaches. Eds. M. Ashraf and P. J. C. Harris (New York: Haworth Press Inc.), 277-300. doi: 10.1201/9781482293609-18

Hu, Y., Han, Y. T., Zhang, K., Zhao, F., Li, Y. J., Zheng, Y., et al. (2016). Identification and expression analysis of heat shock transcription factors in the wild Chinese grapevine (Vitis pseudoreticulata). Plant Physiol. Biochem. 99, 1-10. doi: 10.1016/j.plaphy.2015.11.020

Hua, J., Grisafi, P., Cheng, S. H., and Fink, G. R. (2001). Plant growth homeostasis is controlled by the Arabidopsis BON1 and BAP1 genes. Genes Dev. 15, 2263 2272. doi: 10.1101/gad.918101

Iandolino, A. B., Goes da Silva, F., Lim, H., Choi, H., Williams, L. E., and Cook, D. R. (2004). High-quality RNA, cDNA, and derived EST libraries from grapevine (Vitis vinifera L.). Plant Mol. Biol. Rep. 22, 269-278. doi: $10.1007 /$ bf02773137

Jiang, J. F., Liu, X. N., Liu, C. H., Liu, G. T., Li, S. H., and Wang, L. J. (2017). Integrating omics and alternative splicing reveals insights into grape response to high temperature. Plant Physiol. 173, 1502-1518. doi: 10.1104/pp.16.01305

Kosová, K., Vítámvás, P., Prášil, I. T., and Renaut, J. (2011). Plant proteome changes under abiotic stress-contribution of proteomics studies to understanding plant stress response. J. Proteom 74, 1301-1322. doi: 10.1016/ j.jprot.2011.02.006

Larkindale, J., and Vierling, E. (2008). Core genome responses involved in acclimation to high temperature. Plant Physiol. 146, 748-761. doi: 10.1104/ pp.107.112060

Larkindale, J., Hall, J. D., Knight, M. R., and Vierling, E. (2005). Heat stress phenotypes of Arabidopsis mutants implicate multiple signaling pathways in the acquisition of thermotolerance. Plant Physiol. 138, 882-897. doi: 10.1104/ pp.105.062257

Li, Q. X., Wang, W. Q., Wang, W. L., Zhang, G. Q., Liu, Y., Wang, Y., et al. (2018). Wheat F-box protein gene TaFBA1 is involved in plant tolerance to heat stress. Front. Plant Sci. 9, 521. doi: 10.3389/fpls.2018.00521

Liang, X. Q., Ma, N. N., Wang, G. D., Meng, X., Ai, X. Z., and Meng, Q. W. (2015). Suppression of SINAC1 reduces heat resistance in tomato plants. Biol. Plant 59, 92-98. doi: 10.1007/s10535-014-0477-7

Liu, H. T., Sun, D. Y., and Zhou, R. G. (2010). Ca ${ }^{2+}$ and AtCAM3 are involved in the expression of heat shock protein gene in Arabidopsis. Plant Cell Environ. 28, 1276-1284. doi: 10.1111/j.1365-3040.2005.01365.x 
Liu, G. T., Wang, J. F., Cramer, G., Dai, Z. W., Duan, W., Xu, H. G., et al. (2012). Transcriptomic analysis of grape (Vitis vinifera L.) leaves during and after recovery from heat stress. BMC Plant Biol. 12, 174. doi: 10.1186/1471-2229-12-174

Liu, G. T., Ma, L., Duan, W., Wang, B. C., Li, J. H., Xu, H. G., et al. (2014). Differential proteomic analysis of grapevine leaves by iTRAQ reveals responses to heat stress and subsequent recovery. BMC Plant Biol. 14, 110. doi: 10.1186/1471-2229-14-110

Livak, K. J., and Schmittgen, T. D. (2001). Analysis of relative gene expression data using real-time quantitative PCR and the $2^{-\Delta \Delta C T}$ method. Methods. 25, 402408. doi: 10.1006/meth.2001.1262

Lobell, D. B., Burke, M. B., Tebaldi, C., Mastrandrea, M. D., Falcon, W. P., and Naylor, R. L. (2008). Prioritizing climate change adaptation needs for food security in 2030. Science. 319, 607-610. doi: 10.1126/science.1152339

Lorenz, D. H., Eichhorn, K. W., Bleiholder, H., Klose, R., Meier, U., and Weber, E. (1995). Growth stages of the grapevine: phenological growth stages of the grapevine (Vitis vinifera L. ssp. vinifera)-codes and descriptions according to the extended $\mathrm{BBCH}$ scale. Aust. J. Grape Wine Res. 1, 100-103. doi: 10.1111/j.1755-0238.1995.tb00085.x

Miller, G., Suzuki, N., Rizhsky, L., Hegie, A., Koussevitzky, S., and Mittler, R. (2007). Double mutants deficient in cytosolic and thylakoid ascorbate peroxidase reveal a complex mode of interaction between reactive oxygen species, plant development, and response to abiotic stresses. Plant Physiol. 144, 1777-1785. doi: 10.1104/pp.107.101436

Mittler, R., Vanderauwera, S., Suzuki, N., Miller, G., Tognetti, V. B., Vandepoele, K., et al. (2011). ROS signaling: the new wave? Trends Plant Sci. 16, 300-309. doi: $10.1016 /$ j.tplants.2011.03.007

Moore, K., and Roberts, L. J. (1998). Measurement of lipid peroxidation. Free Radic. Res. 28, 659-671. doi: 10.3109/10715769809065821

Morrell, A. M., Wample, O. L., Mink, G.II, and Ku, M. S. B. (1997). Expression of heat shock proteins in dormant buds of grape (Vitis vinifera L.). Am. J. Enol. Viticult 48, 465-470. doi: 10.1016/S0065-2164(08)70466-0

Peng, S. B., Zhu, Z. G., Zhao, K., Shi, J. L., Yang, Y. Z., He, M. Y., et al. (2013). A novel heat shock transcription factor, VpHsf1, from Chinese Wild Vitis pseudoreticulata is involved in biotic and abiotic stresses. Plant Mol. Biol. Rep. 31, 240-247. doi: 10.1007/s11105-012-0463-1

Pereira, H. S., Delgado, M., Avó, A. P., Barão, A., Serrano, I., and Viegas, W. (2014). Pollen grain development is highly sensitive to temperature stress in Vitis vinifera. Aust. J. Grape Wine Res. 20, 474-484. doi: 10.1111/ajgw.12105

Pillet, J., Egert, A., Pieri, P., Lecourieux, F., Kappel, C., Charon, J., et al. (2012). $V v G O L S 1$ and $V v H s f A 2$ are involved in the heat stress responses in grapevine berries. Plant Cell Physiol. 53, 1776-1792. doi: 10.1093/pcp/pcs121

Qu, A. L., Ding, Y. F., Jiang, Q., and Zhu, C. (2013). Molecular mechanisms of the plant heat stress response. Biochim. Biophys. Res. Com 432, 203-207. doi: 10.1016/j.bbrc.2013.01.104

Rocheta, M., Becker, J. D., Coito, J. L., Carvalho, L., and Amancio, S. (2014). Heat and water stress induce unique transcriptional signatures of heat-shock proteins and transcription factors in grapevine. Funct. Integr. Genomic 14, 135-148. doi: 10.1007/s10142-013-0338-Z

Salazar-Parra, C., Aguirreolea, J., Sanchez-Diaz, M., Irigoyen, J. J., and Morales, F. (2010). Effects of climate change scenarios on Tempranillo grapevine (Vitis vinifera L.) ripening: response to a combination of elevated $\mathrm{CO}_{2}$ and temperature, and moderate drought. Plant Soil 337, 179-191. doi: 10.1007/s11104-010-0514-z

Schöffl, F., Prandl, R., and Reindl, A. (1998). Regulation of the heat-shock response. Plant Physiol. 117, 1135-1141. doi: 10.1104/pp.117.4.1135

Suzuki, N., Miller, G., Morales, J., Shulaev, V., Torres, M. A., and Mittler, R. (2011). Respiratory burst oxidases: The engines of ROS signaling. Curr. Opin. Plant Biol. 14, 691-699. doi: 10.1016/j.pbi.2011.07.014

Szenteleki, K., Ladanyi, M., Gaal, M., Zanathy, G., and Bisztray, G. (2012). Climatic risk factors of Central Hungarian grape growing regions. Appl. Ecol. Environ. Res. 10, 87-105. doi: 10.15666/aeer/1001_087105

Wahid, A., Gelani, S., Ashraf, M., and Foolad, M. (2007). Heat tolerance in plants: An overview. Environ. Exp. Bot. 61, 199-223. doi: 10.1016/j.envexpbot.2007.05.011

Wang, K., Zhong, M., Wu, Y. H., Bai, Z. Y., Liang, Q. Y., Liu, Q. L., et al. (2017). Overexpression of chrysanthemum transcription factor gene DgNAC1 improves the salinity tolerance in chrysanthemum. Plant Cell Rep. 36, 571581. doi: 10.1007/s00299-017-2103-6

Wang, L., Zhao, R. R., Zheng, Y. Y., Chen, L., Li, R., Ma, J. F., et al. (2017). SlMAPK1/2/3 and antioxidant enzymes are associated with $\mathrm{H}_{2} \mathrm{O}_{2}$-induced chilling tolerance in tomato plants. J. Agric. Food Chem. 65, 6812-6820. doi: $10.1021 /$ acs.jafc.7b01685
Wang, X. L., Xu, C. X., Cai, X. F., Wang, Q. H., and Dai, S. J. (2017). Heatresponsive photosynthetic and signaling pathways in plants: insight from proteomics. Int. J. Mol. Sci. 18, 19. doi: 10.3390/ijms18102191

Xin, H. P., Zhu, W., Wang, L. N., Xiang, Y., Fang, L. C., Li, J. T., et al. (2013). Genome wide transcriptional profile analysis of Vitis amurensis and Vitis vinifera in response to cold stress. PloS One 8, e58740. doi: 10.1371/ journal.pone.0058740

Xu, W. R., Li, R. M., Zhang, N. B., Ma, F. L., Jiao, Y. T., and Wang, Z. P. (2014). Transcriptome profiling of Vitis amurensis, an extremely cold-tolerant Chinese wild Vitis species, reveals candidate genes and events that potentially connected to cold stress. Plant Mol. Biol. 86, 527-541. doi: 10.1007/s11103-014-0245-2

Xu, Y., Burgess, P., Zhang, X. Z., and Huang, B. R. (2016). Enhancing cytokinin synthesis by overexpressing ipt alleviated drought inhibition of root growth through activating ROS-scavenging systems in Agrostis stolonifera. J. Exp. Bot. 67, 1979-1992. doi: 10.1093/jxb/erw019

Yang, S. H., and Hua, J. (2004). A haplotype-specific resistance gene regulated by BONZAI1 mediates temperaturea-dependent growth control in Arabidopsis. Plant Cell. 16, 1060-1071. doi: 10.1105/tpc.020479

Yang, H. J., Li, Y. Q., and Hua, J. (2006). The C2 domain protein BAP1 negatively regulates defense responses in Arabidopsis. Plant J. 48, 238-248. doi: 10.1111/ j.1365-313X.2006.02869.x

Yang, H. J., Yang, S. H., Li, Y. Q., and Hua, J. (2007). The Arabidopsis BAP1 and BAP2 genes are general inhibitors of programmed cell death. Plant Physiol. 145, 135-146. doi: 10.1104/pp.107.100800

Zhang, J. H., Huang, W. D., Pan, Q. H., and Liu, Y. P. (2005). Improvement of chilling tolerance and accumulation of heat shock proteins in grape berries (Vitis vinifera cv. Jingxiu) by heat pretreatment. Post Biol. Technol. 38, 80-90. doi: 10.1016/j.postharvbio.2005.05.008

Zhang, W., Zhou, R. G., Gao, Y. J., Zheng, S. Z., Xu, P., Zhang, S. Q., et al. (2009). Molecular and genetic evidence for the key role of AtCaM3 in heat-shock signal transduction in Arabidopsis. Plant Physiol. 149, 1773-1784. doi: 10.1104/ pp.108.13374

Zhang, X., Wang, L., Meng, H., Wen, H., Fan, Y., and Zhao, J. (2011). Maize ABP9 enhances tolerance to multiple stresses in transgenic Arabidopsis by modulating ABA signaling and cellular levels of reactive oxygen species. Plant Mol. Biol. 75, 365-378. doi: 10.1007/s11103-011-9732-x

Zhang, G. K., Xiao, P. L., Hou, L. X., Wang, W. J., Ma, Q., and Liu, X. (2014). Gene cloning and expression analysis of VvBAP1 in Vitis vinifera. Plant Physiol. J. 50, 829-834. doi: 10.13592/j.cnki.ppj.2014.0028

Zhao, D. Y., Shen, L., Fan, B., Liu, K. L., Yu, M. M., Zheng, Y., et al. (2009). Physiological and genetic properties of tomato fruits from 2 cultivars differing in chilling tolerance at cold storage. J. Food Sci. 74, C348-C352. doi: 10.1111/ j.1750-3841.2009.01156.x

Zhao, Q., Zhou, L. J., Liu, J. C., Du, X. X., Asad, M., Huang, F. D., et al. (2018). Relationship of ROS accumulation and superoxide dismutase isozymes in developing anther with floret fertility of rice under heat stress. Plant Physiol. Bioch. 122, 90-101. doi: 10.1016/j.plaphy.2017.11.009

Zhou, J., Wang, J., Yu, J. Q., and Chen, Z. X. (2014). Role and regulation of autophagy in heat stress responses of tomato plants. Front. Plant Sci. 5, 174. doi: $10.3389 /$ fpls.2014.00174

Zhu, Y., Yang, H. J., Mang, H. G., and Hua, J. (2011). Induction of BAP1 by a moderate decrease in temperature is mediated by ICE1 in Arabidopsis. Plant Physiol. 155, 580-588. doi: 10.1104/pp.110.169466

Zhuang, J., Zhang, J., Hou, X. L., Wang, F., and Xiong, A. S. (2014). Transcriptomic, proteomic, metabolomic and functional genomic approaches for the study of abiotic stress in vegetable crops. Crit. Rev. Plant Sci. 33, 225-237. doi: 10.1080/07352689.2014.870420

Conflict of Interest: The authors declare that the research was conducted in the absence of any commercial or financial relationships that could be construed as a potential conflict of interest.

Copyright (c) $2020 \mathrm{Ye}, \mathrm{Yu}$, Zhang, Hou and Liu. This is an open-access article distributed under the terms of the Creative Commons Attribution License (CC BY). The use, distribution or reproduction in other forums is permitted, provided the original author(s) and the copyright owner(s) are credited and that the original publication in this journal is cited, in accordance with accepted academic practice. No use, distribution or reproduction is permitted which does not comply with these terms. 